Instituto Internacional de Investigación y Desarrollo Tecnológico Educativo INDTEC, C.A.

DOI: https://doi.org/10.29394/scientific.issn.2542-2987.2017.2.6.5.92-112

OAI-PMH: http://www.indteca.com/ojs/index.php/Revista Scientific/oai

\title{
Planeación de la Educación Ambiental en el Ámbito Universitario: Una Mirada Transdisciplinaria
}

\author{
Autora: Emilia Montesinos de Rodríguez \\ Universidad Fermín Toro, UFT \\ emilia.montesinos@gmail.com \\ Mérida, Venezuela
}

\section{Resumen}

La investigación se dirige a producir una reflexión ontoepistémica sobre la planeación de la Educación Ambiental en el ámbito universitario con enfoque transdisciplinar. Se abordó con el paradigma interpretativo, métodos hermenéuticos y dialectico e investigación documental. La información recopilada da cuenta de una fiabilidad hermenéutica, recurriendo a la categorización, estructuración, contrastación y teorización. Los resultados reflejan que en la educación universitaria han predominado los postulados centrados en la enseñanza unidireccional, fragmentada, disciplinar, con parcelamiento de los saberes. Desde el diseño de los programas hasta la praxis académica, la construcción del conocimiento se hace de forma aislada con tendencia a formar estudiantes poco motivados a interactuar en el escenario donde se desenvuelven e indiferentes ante los problemas socio ambientales. No se asume la planeación de la Educación Ambiental como forma activa de articular la praxis educativa con la situación problemática del medio ambiente que hoy enfrenta la humanidad. En conclusión, la planeación de la Educación Ambiental en el ámbito universitario debe girar en torno a la complementariedad entre las diversas áreas del conocimiento, donde se logre una actitud reflexiva y trascendente para la enseñanza de orden superior, en la cual la realidad ambiental y social se conciba como fenómenos cambiantes y complejos.

Palabras clave: planeación; educación ambiental; ámbito universitario; transdisciplinariedad. 


\title{
Planning of Environmental Education in the University Field: A Transdisciplinary Look
}

\begin{abstract}
The research is directed to produce an ontoepistemic reflection about Environmental Education planning at the university field with a transdisciplinary approach. It was focused with the interpretative paradigm, hermeneutic and dialectical methods and documentary research. The information collected gives a hermeneutical reliability, categorization, structuring, contrasting and theorizing. The results reflect that university education have predominated postulates centered on unidirectional, fragmented, disciplinary teaching, with sort of the knowledge. From programs design to academic praxis, the construction of knowledge is done in an isolated way with tendency to prepare students lack motivated to interact in the stage where they develop and indifferent to socio-environmental problems. Planning of Environmental Education is not assumed as an active way to linking educational praxis with the problematic environment situation that humanity faces today. The results indicated that the Environmental Education planning at the university field should revolve around complementarity with different areas of knowledge, a reflexive and transcendent attitude for higher education, in which environmental and social reality is conceived as changing and complexes phenomena.
\end{abstract}

Keywords: planning; environmental education; university environment; transdisciplinarity. 


\section{Introducción}

El paso de la modernidad a la postmodernidad demanda cambios del paradigma educativo amparado hasta ahora en la fragmentación de la realidad, el cientificismo, la ciencia mecanicista y aislado del contexto de orden planetario; en este sentido, la Universidad como espacio formativo se mantiene en los planteamientos teóricos y la parcelación de saberes en micro disciplinas o de manera disciplinaria.

Desde la perspectiva hologramática de la complejidad, la realidad no se debe particularizar sino, estudiar las partes para entender el todo, tampoco el todo sin entender las partes que lo componen, así como manejar todas esas partes desde el punto de vista del conocimiento que las caracteriza, la idea del holograma trasciende a que la realidad no está hecha de partes, como lo suponen los reduccionistas, ni tampoco de totalidades, como afirman los holistas. (Morín, 2011, pág. 107)

Ahora bien, las instituciones universitarias como sistemas abiertos están distantes a vincularse con el contexto social, establecer relación permanente con el medio ambiente, compartir la incertidumbre generada por los eventos naturales, la violencia, el hambre, las pandemias y epidemias, entre otros; comprendiendo la complejidad de interacciones y la resignificación del pensamiento en los individuos, como respuestas a las necesidades sociales.

En este orden de ideas, en los docentes se avista poco interés por la planificación dirigida hacia una educación holista en la cual se perciba el universo como una comunidad de sujetos, las estrategias de enseñanza no permiten el desarrollo de habilidades ni cambios de comportamientos y redimensión de las actitudes, para establecer relaciones entre conocimientos previos y nuevas adquisiciones, por ende, interactuar en procesos complejos propios de la naturaleza humana. 
Para cumplir este reto, es necesario tomar en cuenta el talento humano que conforma las aulas universitarias y su potencial creativo, relacionado con el desarrollo de actitudes y valores que tenga como prioridad la relación de la sociedad con su entorno, con el fin de procurar un desarrollo humano sostenible, que pueda garantizar el soporte físico y biológico sobre el cual se sustenta. (Azuaje, 2011, pág. 130)

Los aspectos anteriormente mencionados, corroboran la importancia signada a la planeación educativa, para que exista correspondencia entre la teoría y la práctica que se ejecuta, proponer caminos de actuación, responder a los modelos siempre cambiantes; realidad que da cuenta de la necesidad de una mayor profundización en el tema, a fin de propiciar el interés, la participación, el compromiso del docente universitario, los estudiantes, la institución con el planeta.

Con respecto a la planeación Goodstein, Nolan y Pfeiffer (2001), señalan lo siguiente:

...es el proceso de establecer objetivos y escoger el medio más apropiado para el logro de los mismos antes de emprender la acción (...) La planeación se anticipa a la toma de decisiones. Es un proceso de decidir antes de que se requiera la actuación. (pág. 5).

En este sentido, la planificación educativa debe servir de direccionamiento a la Educación Ambiental, donde aprender implique una connotación especial, como proceso de la conciencia humana en lo afectivo, físico, social y espiritual; atendiendo a la cosmovisión sobre el entorno, donde la interacción permita la comprensión socio natural y el compromiso con el ambiente. En torno a estas ideas, es necesario asegurar el espacio de la interconexión entre las disciplinas, lo que exige un pensamiento transdisciplinar, transversal, una reflexión que relacione la parte y el todo, el micro y el macro, lo singular y lo universal; se concreta en la medida en que 
se desarrolla la interdisciplinariedad mediante la integración de las diferentes disciplinas, dado que su expansión adquiere carácter transdisciplinario cuando se genera un nuevo conocimiento. (Magallanes, 2014, pág. 119).

De ese modo, la planeación basada en la transdisciplinariedad es de carácter transversal, pues los conceptos y procedimientos que se presentan a los estudiantes deben ser organizados globalmente y compartidos por varias disciplinas; el docente universitario tiene la iniciativa de incorporar temas y desarrollar actividades de naturaleza local, así como de propiciar articulaciones con otras áreas de conocimiento y de la realidad del estudiante.

En esencia la apertura hacia una cultura ambientalista demanda que la planeación transdisciplinar tenga como punto de partida las siguientes interrogantes: ¿de qué manera producir una reflexión ontoepistémica sobre planeación de la educación ambiental en el ámbito universitario con una mirada transdisciplinaria? ¿Cuáles son los fundamentos teóricos de la planeación de Educación Ambiental se desarrollan en el ámbito universitario? ¿Cuáles son los principios teóricos que rigen la transdisciplinariedad en Educación Ambiental?

Como efecto de estas interrogantes surgieron los siguientes objetivos, en cuanto al general, producir una reflexión ontoepistémica sobre planeación de la educación ambiental en el ámbito universitario con una mirada transdisciplinaria; con relación a los específicos: Comprender los fundamentos teóricos de la planeación de Educación Ambiental se desarrollan en el ámbito universitario y, Develar los principios teóricos que rigen la transdisciplinariedad en Educación Ambiental.

El resultado de los mismos se alcanzó con la aplicación metodológica comprendida en el paradigma interpretativo y la investigación documental con los métodos hermenéutico - dialéctico; lo cual trascendió a la interpretación y el análisis de los referentes teóricos considerados y por ende imprimió el carácter de credibilidad de la información. Con este estudio, se pretende la 
producción de una reflexión ontoepistémica sobre la planeación de la educación ambiental con una mirada transdisciplinaria, dirigida a concienciar a los actores educativos de la universidad sobre la evidente responsabilidad de asistir la problemática ambiental que presenta el planeta y por ende, contribuir a fomentar entre las personas el amor y respeto hacia el medio; se estructuró en las siguientes secciones: el resumen, introducción, desarrollo teórico, metodología, resultados conclusiones y referencias bibliográficas.

\section{Teorías y Conceptos}

En esta investigación se recopilan los datos de interés mediante la revisión documental de los fundamentos teóricos acerca de la planeación educativa, la educación ambiental en el ámbito universitario y la transdisciplinariedad; los resultados constituyen un aporte al conocimiento a fin de hacer frente a los cambios que exige la sociedad actual ante las condiciones del devenir entre la modernidad y la postmodernidad.

\subsection{Planeación educativa}

La planificación es un proceso para la toma de decisiones, con el fin de alcanzar un futuro deseado, teniendo en cuenta la situación actual, factores internos y externos puedan influir en la consecución de los objetivos en la transformación de una determinada realidad; por ende, es indispensable como recurso de desarrollo de la sociedad en los procesos económicos, educativos, culturales, científicos, tecnológicos y políticos.

En efecto, es la primera etapa del proceso administrativo, cuya función se plantea en determinar cuáles son los objetivos deseados y lo que debe hacerse para alcanzarlos de manera adecuada; históricamente emerge en el contexto de la administración con las teorías de Tylor (1911) y Fayol (1916), quienes presentan dos aproximaciones, la definición del objetivo o logicidad como marca de una trayectoria con secuencia direccional, y, la sistematicidad 
del proceso, que funciona en un sistema con sinergia estratégica. (Chiavenato, 2007, pág. 48)

En este sentido, planificar es fijar cursos de acción para alcanzar determinados objetivos, mediante el uso efectivo de recursos y procedimientos, para lo cual se debe considerar el conocimiento de la realidad. Otros supuestos básicos, son el estructuralismo reconocido por Piaget, el enfoque de sistemas por el CENDES-UCV, la teoría política con las luchas sociales y las formas de poder; igualmente se reconoce la temática orientada a propiciar el cambio de paradigmas en la planificación con la aparición de la informática. (Corredor, 2001, pág. 20-34)

Generalmente se confunde planificación con plan, programa y proyecto, sin embargo, en éste trabajo, se distinguen como los niveles operativos de la planificación con alcances muy diferentes entre sí a saber: el primero, hace referencia a lineamientos de carácter global, el segundo a un conjunto integrado de proyectos, y, el tercero se expresa en un conjunto de actividades, las cuales se reconocen como el medio de intervención sobre la realidad para alcanzar las metas y objetivos; otro aspecto a considerar son las tareas y las acciones que tienen el máximo grado de especificidad, forma parte de la vida cotidiana cuando se tiene una intención por concretar.

En este orden de ideas, existe otra clasificación de la planeación por tiempo invertido o modelo educativo: a) Anual, es un diseño que contempla los aprendizajes que se espera lograr durante todo un año de clases en una institución educativa; b) Unidad didáctica, corresponde a que cada docente decide realizar del programa, en el cual considera el tiempo necesario para que los estudiantes logren un aprendizaje determinado; y, c) De clase, corresponde al trabajo personal del docente para preparar de forma detallada cada una de sus actividades, que le permita cumplir con el proceso de enseñanza y aprendizaje. (Ander-Egg, 1996, pág. 23). 
Cabe señalar que en estos niveles supone un principio de organización descentralizado y mayor participación del docente, en la elaboración del proyecto educativo y curricular de la Universidad; es evidente por lo tanto, que la planificación no es exclusiva de ningún nivel del sistema educativo, pues en todas las instituciones se planifica, ejecuta y controla, éstos mecanismos buscan la coordinación de las actuaciones que demanda un proceso educativo de calidad.

A partir de este discernimiento, la planeación educativa es entendida como un proceso sistemático, continuo y abierto, en el cual resaltan los siguientes principios: a) El proceso de planificación puede convertirse en un instrumento de innovación, pues el cambio suscita nuevas estrategias; b) Lo anterior implica participación de los usuarios y coherencia en las actuaciones. Y, c) Ordenación y aprovechamiento de los medios existentes con vistas a la consecución de los objetivos planteados en función de la realidad educativa. (Gairín, 2000 pág. 258-259).

Cabe destacar que, en el abordaje de la planificación en una realidad compleja y sistémica, es necesario que las partes den cuenta del todo, de esta relación surge la complementariedad de las disciplinas; habida cuenta de que la transdisciplinariedad en el ámbito del pensamiento complejo tiende a considerar los fenómenos que se entretejen de manera dinámica, dando como resultado la ilación transversal de necesidades sociales y contenidos actitudinales preferiblemente.

\subsection{Educación Ambiental}

Tradicionalmente, el propósito de la Educación Ambiental es la de transmisor-de conocimientos, formar valores, desarrollar competencias y comportamientos que puedan favorecer la comprensión y la solución de los problemas ambientales. Debe ser un proceso permanente que involucre a 
todos y permita un análisis de los principales problemas que afectan al ambiente y la identificación de posibles soluciones a los mismos.

Por ello, con la enseñanza de la Educación Ambiental se aspira a que el ser humano comprenda la naturaleza compleja del ambiente resultante de la interacción de los componentes biológicos, físicos, sociales y culturales; es la contribución para formar individuos, que su accionar contribuya al desarrollo de las comunidades que habitan.

Por lo tanto, la Educación Ambiental debe involucrarse como un proceso que produce cambios en el pensamiento del estudiante en lo conceptual, actitudinal y valores, así como, en la conducta de grupos comunitarios o sociales; en este orden de ideas, es conveniente describir la definición de la Educación Ambiental desde la perspectiva de Zimmermann (2013):

(...) busca tratar temas y problemas ambientales en el aula o en taller, sobre todo en entornos naturales y urbanos (...) acción tendiente al cambio de actitudes de los educandos, con el propósito de volver sus conductas más relevantes en cuanto a responsabilidad y valoración ecológica, frente a la no contaminación, el reciclaje de desechos sólidos mejoras en los espacios naturales y construidos. (pág. 33)

Es un proceso que debe realizarse en todo el período educativo, es decir o sea durante toda la vida, por lo cual debe iniciarse a temprana edad y continuar en la enseñanza primaria, secundaria y universitaria, con prosecución en la educación formal e informal. Es importante aclarar que la apreciación de los aspectos conceptuales está referida a conocer el ambiente para tomar conciencia de la situación y de allí adoptar decisiones acertadas y responsable; por otro lado, la Educación Ambiental trata de ver al ambiente como un sistema en el que interactúa el hombre y cultura con la naturaleza; de ahí la necesidad de desarrollar una visión sistémica que supone una aportación a otras disciplinas. 
Como todo proceso educativo, la Educación Ambiental también tiene sus objetivos, establece prioridad en la práctica para ello es vital la didáctica, las estrategias y recursos; pues se ha pretendido su enseñanza desde el enfoque sistémico buscando la integración las partes para formar una unidad de manera que un cambio en alguna de ellas afecta a las demás; no obstante, en muchas ocasiones desde esta realidad se analiza la problemática del planeta intentando desarrollar actitudes y aptitudes pero no se promueve la participación en los educandos.

En este sentido, se pretende un desarrollo integral en los estudiantes para ello se conjuga la adquisición de conocimientos propios de la especialidad de estudio y la interrelación con otras disciplinas, además de la adquisición de habilidades, actitudes y valores; por lo que la planeación transdisciplinaria se ventila como una vía expedita para la enseñanza de la Educación Ambiental en el contexto universitario fomentando que lo aprendido se comprenda y no se memorice al afrontar situaciones de la realidad.

\subsection{Transdisciplinariedad}

El conocimiento transdisciplinar proviene como un requerimiento de la sociedad, ligado directamente a lo social, a lo ecológico, y a la educación, donde las posibles soluciones necesitan de la producción del conocimiento, lo cual debe producir cambios significativos; para ello se requiere de un escenario de que permitan enfocar la realidad múltiple tal cual y como existe en el pensamiento del individuo.

De este modo, la transdisciplinariedad nace del aporte de las disciplinas a las que interrelaciona y supera; entonces la mirada "trans" de la realidad proyecta un pensamiento de globalidad, por lo puede concebirse como un proceso cognitivo y afectivo, aunado a los aspectos socioculturales, históricos y políticos en la producción de conocimientos multidimensionales, no parcelados ni reduccionistas. (Figueredo, 2014, pág. 91); en efecto, la 
transdisciplinariedad es un principio epistemológico de reorganización del saber, que facilita la comprensión de la realidad y permite la migración de conceptos entre diferentes campos del conocimiento.

La búsqueda de una actitud transdisciplinaria implica una ventana a la transformación de la educación que no requiere sólo de información y de conocimientos, sino, de participación y apertura hacia sectores de la sociedad y el ambiente buscando con ello una resignificación de lo humano y el universo, es decir el devenir planetario de la humanidad, lejos de convertirse pragmáticamente en religión, filosofía o ciencia.

Por su naturaleza, la Universidad se debe plantear entre sus objetivos salir del parcelamiento y fragmentación de los saberes evolucionando hacia el estudio de lo universal; Locarno, citado por Martínez (2009), considera “...Que es necesario hacer penetrar el pensamiento complejo y la transdisciplinariedad dentro de las estructuras y programas de la universidad del mañana" (pág. 24); pues el mundo se construye de relaciones a nivel global donde los fenómenos socio-educativos son interdependientes.

De igual forma, el proceso activo de enseñanza en el cual se desenvuelve el docente universitario, se enmarcarse en un mundo de complejidad, por la apertura de áreas abiertas a las nuevas formas de dosificar el conocimiento, con desafíos pedagógicos de coherencia social, para el análisis y reflexión de los actuales gestores del conocimiento; pues existe una tendencia en el ámbito universitario que le dan la connotación de globalizados, por el esfuerzo, para proyectar la necesidad del cambio de pensamiento docente, por su manera de observar y transformar la realidad. (Maldonado, 2017, pág. 230).

\section{Metodología}

El camino metodológico de esta indagación se situó en el paradigma Interpretativo, asumiendo la investigación documental y lo hermenéutico- 
dialectico, como el método que permite la reflexión ontoepistémica sobre la Planeación de la Educación Ambiental en el ámbito universitario y su vinculación con el enfoque de la transdisciplinariedad.

Martínez, V. (2013), argumenta lo siguiente:

(...) El paradigma interpretativo en vías de consolidación, emerge a partir del reconocimiento de la importancia de los aspectos simbólicos y significativos de la vida social y del lenguaje en la producción y reproducción del mundo, (...) Se apoya en sistemas filosóficos y se operacionaliza mediante un sistema de investigación hermenéutico. (pág. 1)

Es el proceso basado en el naturalismo, donde prevalecen las opiniones, por enfocarse en la producción axiológica en función de la experiencia, partiendo del conocimiento del fenómeno dentro del contexto social, de manera interpretativa, para llegar a la teorización; tiene su propia manera de entender la realidad partir de fundamentos ontológicos, epistemológicos y metodológicos.

Asumir una perspectiva interpretativa implica un esfuerzo de comprensión, entendido como la captación a través de la interpretación y el dialogo del sentido de lo que otros quieren decir con sus palabras, silencio, gestos o acciones. (Rojas, 2014 pág. 46); se esboza así, la planificación en educación ambiental como una situación socioeducativa abordada desde el conocimiento orientado hacia los transdisciplinario, sistémico y complejo, con predominio del dialogo en vista del ritmo acelerado de los cambios a nivel mundial.

En este orden de ideas, la investigación documental proporciona una visión global de la realidad por investigar y permite establecer categorías conceptuales para determinar el carácter teórico y práctico de la misma.

Palella y Martins (2016a), señalan lo siguiente:

Entendemos la investigación documental como un proceso de búsqueda que se realiza en fuentes, con el objeto de recoger 
información, organizarla, describirla e interpretarla de acuerdo con ciertos procedimientos que garanticen confiabilidad y objetividad en la presentación de sus resultados. (...) (pág. 100).

Por cuanto, la fuente principal de información está integrada por documentos y el interés del investigador consiste en analizarlos como situaciones o instrumentos que brindan información sobre la situación objeto de estudio.

De igual forma, para la construcción teórica se perfiló el diseño bibliográfico, referenciado por Palella y Martins (2016b), como:

Se fundamenta en la revisión sistemática, rigurosa y profunda de material documental de cualquier clase (...), el investigador utiliza documentos; los recolecta, selecciona, analiza y presenta resultados coherentes. Es un proceso que se realiza en forma ordenada y con objetivos precisos, con la finalidad de fundamentar la construcción de conocimientos. (pág. 96)

En efecto, a las diferentes fuentes bibliográficas se les aplicó una revisión sistemática permitiendo la búsqueda de la información para producir la reflexión ontoepistémica; una vez depurada y seleccionada, se corroboró la pertinencia con los constructos, se aplicaron las técnicas de resumen y el análisis de contenido. El diseño de la investigación viene dado por la revisión exhaustiva de las diferentes fuentes de información, tales como documentos, informes, estudios, ponencias, leyes, normas y bibliografía relacionada con los constructos de estudio, dignos de la hermeneusis como el arte de interpretar, explicar y traducir del texto objetivamente las acciones humanas.

El método hermenéutico es adecuado para el estudio de la acción humana, donde se involucra la interpretación y el análisis de fenómenos significativos, tomando en cuenta la necesidad que tiene el individuo de 
descifrar las diversas situaciones acontecidas en la vida diaria y en el entorno que los rodea.

Para Dilthey, citado por Martínez (2006), la hermenéutica:

(...), es el proceso por medio del cual conocemos la vida psíquica con la ayuda de signos sensibles que son su manifestación..., tendría como misión descubrir los significados de las cosas, interpretar lo mejor posible las palabras, los escritos, los textos y los gestos, así como cualquier acto u obra (pág. 119).

Por su parte el método dialéctico, proporciona la posibilidad de comprender los diversos fenómenos y leyes que afronta la realidad misma de las cosas, funciona como uso del razonamiento al confrontar diversas posiciones, y extraer de ellas la verdad mediante la discusión de opiniones, al descubrir las contradicciones en las argumentaciones del interlocutor.

Al respecto, Gadamer (1993), expresa que:

(...), el intentar desarrollar un concepto de conocimiento y de verdad que responda al conjunto de la experiencia hermenéutica se está apuntando hacia una nueva forma heurística, que camina en la dirección de la transdisciplinariedad, que, como vivencia, queda integrada en el todo de la vida y, por lo tanto, el todo se hace también presente en ella es un auténtico conocimiento (pág. 78).

El método hermenéutico-dialéctico se utiliza en todo momento consciente 0 inconsciente, pues la mente humana es por naturaleza, interpretativa en vista de que le da significado a lo observa; permite la comprensión y entendimiento, producto de la reflexión dialógica con los autores citados, generando el nuevo enfoque que caracterizó las categorías; de allí que las unidades de estudio estuvieron representadas por los diferentes materiales referenciales, es decir, las ideas se descompusieron en unidades, lo más pequeñas posible, siendo reagrupadas en un concepto que permitió dar otra orientación, tomando en cuenta los componentes ontológicos y 
gnoseológicos de los autores citados y desde la realidad de la investigadora. Hurtado (2014), resalta que: "las unidades de estudio se deben definir de tal modo que a través de ellas se puedan dar una respuesta completa y no parcial a la interrogante de la investigación". (pág. 152).

En este orden de ideas la reducción de la información recabada se realizó con la práctica las siguientes fases: categorización, contrastación y teorización, estas fases permitieron darle el tratamiento adecuado por ser propios del paradigma de investigación interpretativo. De acuerdo con la visión de Martínez (2002), describir categorías es “...clasificar las partes significativas, de ir constantemente diseñando y rediseñando, integrando y reintegrando el todo y las partes, a medida que se revisa el material y va emergiendo el significado de cada sector, evento, hecho o dato" (pág. 71). Es el surgimiento de unidades apriorísticas para reordenarlas desde su entramado de unidades emergentes con nuevos significados.

De igual forma, la información recopilada permitió la fiabilidad de manera hermenéutica y dialógica, recurriendo a la comparación de los componentes ontológicos, contrastando los resultados mediante la triangulación, que permitieron la interpretación del pensamiento de los autores citados, partiendo de la descripción de categorías y subcategorías hasta la teorización.

Respecto a la teorización, Ruiz, (2003), indica lo siguiente: (...) la estrategia de una investigación cualitativa va orientada a descubrir, captar y comprender una teoría, una explicación un significado (...) (pág. 57); en este sentido, la reflexión ontoepistémica emergió a partir del contraste de la información recabada en función de las vivencias de la investigadora, con ideas y frases implícitas en el del estudio realizado a los constructos planificación, transdisciplinariedad y Educación Ambiental en el ámbito universitario. 


\section{Resultados y Discusiones}

Los resultados de la investigación dan cuenta de un contexto universitario, en el cual la planeación de la Educación Ambiental continúa con apropiación disciplinaria de los saberes desde la organización administrativa de facultades, programas y departamentos, sin ningún vínculo entre ellos; mientras que la dinámica exige un trabajo académico transdisciplinar, en el cual se permita el conocimiento, experiencias y la comprensión de las realidades. La universidad tiene una gran responsabilidad para propiciar este cambio, tomando en cuenta una educación de orden superior que involucre el desarrollo cultural, socioeconómico y ecológico sostenible de los individuos

En éste orden de ideas, las universidades presentan una insuficiente oferta de programas educativos que aborden temas sobre las transformaciones en el medio ambiente ocasionados por las actividades humanas tales como el calentamiento global del planeta, la destrucción de la capa de ozono, la sobrepoblación humana, los cambios climáticos, entre otros; se hace referencia a planes de estudio estáticos, con metodología de enseñanza y aprendizaje que no promuevan la activa participación de los estudiantes.

En las instituciones universitarias también resaltan deficiencias centradas en planificar para la búsqueda exclusiva de una mayor eficiencia administrativa, con tendencia a considerar que los fines y los medios de que se trata están dados y son fijos, o por el contrario, que deben buscarse y son variables; ésta situación se reduce a la simple búsqueda de una mejor forma de organizar, sin cuestionar si éstos son los más dignos de ser perseguidos, y los medios los más conducentes a ello. Por lo tanto, a partir de la planeación transdisciplinaria la institución educativa debe establecer una relación sistémica como un proceso para plantear y resolver problemas complejos, donde se conciba el accionar del docente universitario como un conjunto de habilidades que le permiten a los estudiantes el aprendizaje de Educación 
Ambiental en un entorno de constantes cambios, incorporando la interrelación entre sus actores.

Cabe señalar, que el enfoque transdisciplinario se inicia desde que el sujeto comienza a interpretar la realidad e intenta desarrollar conocimientos en correspondencia al conjunto de experiencias, a partir de las cuales extrae la nueva información que está en constante interacción y complementariedad, para contextualizar y globalizar los saberes; así, la transdisciplinariedad es una inquietud del presente ante la especificidad del crecimiento de los saberes en el que se generan desafíos para atender los problemas del contexto, como un medio de establecer la justicia y el bienestar social del individuo.

Entonces, la planeación transdisciplinar es proyectar sobre una actividad a realizar para obtener los mejores resultados con el mínimo de tiempo y de recursos, este proceso de transformación es complejo, por cuanto sobre él confluyen numerosos y diversos factores como la búsqueda de las mejores condiciones para lograr el aprendizaje, el nivel de desarrollo de los estudiantes, la naturaleza de los conocimientos, las condiciones del contexto y las necesidades individuales y colectivas. Es decir, la planeación no resolverá todos los problemas que tenemos, pero tampoco será una herramienta inútil en manos de profesionales con voluntad para dar sentido a sus actividades vitales, entre las que obviamente se encuentra la educativa.

Se discurre que, la producción de un pensamiento transdisciplinar de la educación ambiental, supone reflexionar sobre la panorámica actual que permita un cambio de paradigma con formas de conocimiento referidas a lo creativo y a lo emocional, aportando elementos novedosos a la planeación educativa; habida cuenta, de que la transdisciplinariedad nos ayuda a entender que las propuestas didácticas no deben apoyarse en una planificación positivista desde un pensamiento determinista y reduccionista, y en una metodología que fragmenta el conocimiento y la realidad. 


\section{Reflexiones}

La planeación, es el proceso mediante el cual una institución vislumbra su futuro y desarrolla las operaciones convenientes para lograrlo, es un instrumento necesario para el establecimiento de políticas, objetivos, redacción de programas, entre otros; de esta manera, se convierte en una secuencia sistemática de toma de decisiones; por su parte, el enfoque transdisciplinario se vincula estrechamente con la planeación educativa del ámbito universitario, en vista de que permite iniciar cambios y adaptarlos a las necesidades del entorno, conformando redes de conocimientos.

En este sentido, se considera la transdisciplinariedad como una vía hacia la fusión de disciplinas en una visión incluyente habida cuenta de que relaciona las ciencias exactas con las sociales, rompe con la parcelación del conocimiento por lo cual favorece el pensamiento holístico; de igual forma, implica el manejo de conceptos, métodos y categorías de diferentes áreas del conocimiento, para el tratamiento de problemáticas socio ambientales.

El reto es encontrar las bases de una educación capaz de promover un desarrollo humano integral, para lo cual la Educación Ambiental universitaria se vislumbra como una contribución esencial de interrelación con la sociedad, la confluencia de saberes con el desarrollo de programas orientados a mejorar las relaciones del ser humano con el cosmos.

\section{Referencias}

Ander-Egg, E. (1996). La Planificación educativa. Conceptos, métodos, estrategias y técnicas para educadores. Buenos Aires-Argentina: Magisterio del Rio Plata.

Azuaje, F. (2011). La Dimensión Ambiental en el Contexto Educativo Universitario. Revista Científica Digital del Centro de Investigación y Estudios Gerenciales. Barquisimeto, Venezuela. 1(4). Recuperado de: 
http://www.grupocieg.org/archivos revista/1-4\%20(129-

138)\%20Azuaje\%20rcieg\%20mayo\%2011 articulo id33.pdf

Chiavenato, I. (2007). Introducción a la Teoría General de la

Administración. México: McGraw-Hill.

Corredor, J. (2001). La Planificación Estratégica. Bases teóricas para su aplicación. Caracas, Venezuela: Vadell Hermanos Editores, C.

Figueredo, X. (2014). Transcomplejidad en el lenguaje matemático. Arjé

Revista de Postgrado FACE-UC. 6(10). julio-diciembre 2014 /87-95.

Recuperado de: http://arje.bc.uc.edu.ve/ari15/ari1e15.pdf

Gadamer H. (1993). Verdad y método: fundamentos de una hermenéutica

filosófica. Salamanca, España: Sígueme.

Gairín, J. (2000). La organización escolar: contexto y texto de actuación.

Caracas- República Bolivariana de Venezuela: La Muralla.

Goodstein, L. Nolan, T., y Pfeiffer, J. (2001). Planeación Estratégica Aplicada. Santafé de Bogotá-Colombia: McGraw-Hill.

Hurtado, J. (2014). Metodología de la investigación holística. Caracas, Venezuela: Sypal.

Magallanes, N. (2012). La educación ambiental desde la perspectiva compleja. Una visión Interdisciplinaria y Transdisciplinaria. Arjé Revista de Postgrado FACE-UC. 6(15). julio-diciembre 2014 / 111-120. Recuperado de: http://arje.bc.uc.edu.ve/ari15/arj1e15.pdf

Maldonado, J. (2017). La Hologramática en la Didáctica Matemática Universitaria. Revista Scientific, 215-234. Recuperado de:

https://doi.org/10.29394/scientific.issn.2542-2987.2017.0.0.12.215-234 Martínez, M. (2002). La investigación cualitativa etnográfica en educación: manual teórico práctico. México: Trillas.

Martínez, M. (2006). Comportamiento Humano. Nuevos Métodos de Investigación. México: Trillas.

Martínez, M. (2009). Epistemología y Metodología Cualitativa en las 
Ciencias sociales. México: Trillas

Martínez, V. (2013). Paradigmas de investigación: Manual multimedia para el desarrollo de trabajos de investigación. Una visión desde la epistemología dialéctico-crítica. Recuperado de:

http://www.pics.uson.mx/wp-

content/uploads/2013/10/7 Paradigmas de investigacion 2013.pdf

Morín, E. (2011). Introducción al Pensamiento Complejo. Barcelona, España: Gedisa.

Palella, S., y Martins, F. (2016a,b). Metodología de la investigación cuantitativa. Caracas, Venezuela: FEDUPEL.

Rojas de E. (2014). Investigación cualitativa, fundamentos y praxis. Caracas, Venezuela: FEDUPEL.

Ruiz, O. (2003). Metodología de la investigación cualitativa. Bilbao, España: Universidad de Deusto.

Zimmermann, M. (2013). Pedagogía Ambiental para el planeta en emergencia. Bogotá: Eco ediciones. 


\section{Emilia Montesinos de Rodríguez}

e-mail: emilia.montesinos@gmail.com

Nacida en Mérida, Venezuela. Geógrafo. Universidad

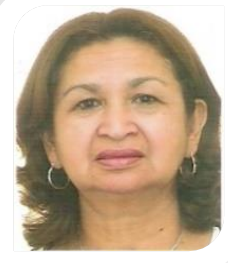
de Los Andes. Mérida. Magister Scientiarum en Educación Superior. Mención Docencia Universitaria. Universidad Fermín Toro 2001. Doctorando en Ciencias de la Educación. Universidad Fermín Toro (2013-2017). Facilitadora de las unidades curriculares: Proyecto I y II, Investigación Evaluativa, Planificación Educativa, Planificación Estratégica, Universidad Valle de Momboy (UVM) 2002-2017. Educación Ambiental e Investigación Educativa (Pregrado). Investigación Cualitativa, Seminario de Investigación I y II (Postgrado) Universidad Pedagógica Experimental Libertador (UPEL) Núcleo Mérida, 2010-2017. Facilitadora Curso de Formación de Tutores: UVM 2009-2017. Ponente del tema Investigación Evaluativa. En: Jornadas Sobre Investigación Educativa UPEL 2012.

El contenido de este manuscrito se difunde bajo una Licencia de Creative Commons ReconocimientoNoComercial-Compartirlgual 4.0 Internacional 